\title{
Clinical Accuracy of a Customized Stereotactic Platform for Deep Brain Stimulation after Accounting for Brain Shift
}

\author{
Pierre-François D'Haese ${ }^{a} \quad$ Srivatsan Pallavaram ${ }^{a}$ Peter E. Konrad ${ }^{b}$ \\ Joseph Neimat ${ }^{b} \quad$ J. Michael Fitzpatrick ${ }^{a}$ Benoit M. Dawant ${ }^{a}$ \\ a Department of Electrical Engineering and Computer Science, Vanderbilt University, and \\ ${ }^{b}$ Department of Neurosurgery, Vanderbilt University Medical Center, Nashville, Tenn., USA
}

\section{Key Words}

Deep brain stimulation - Brain shift - Electrode placement error $\cdot$ Frame accuracy

\begin{abstract}
Previous studies have evaluated the accuracy of several approaches for the placement of electrodes for deep brain stimulation. In this paper, we present a strategy to minimize the effect of brain shift on the estimation of the electrode placement error (EPE) for a stereotactic platform in the absence of intraoperative imaging data, and we apply it to the StarFix microTargeting ${ }^{\circledR}$ Platform (FHC Inc., Bowdoin, Me., USA). This method involves comparing the intraoperative stereotactic coordinates of the implant with its position in the postoperative CT images in a population for which the effect of brain shift is minimal. The study we have conducted on 75 patients demonstrates that the EPE is overestimated at least by about $60 \%$ if brain shift is not taken into account, and shows a clinical accuracy of $1.24 \pm 0.37 \mathrm{~mm}$ for the StarFix frame, which is similar to the reported $G$ frame accuracy and better than the reported Nexframe accuracy $(2.5 \pm 1.4$ $\mathrm{mm}$ ) [Stereotact Funct Neurosurg 2007;85:235-242].
\end{abstract}

Copyright ๑ 2010 S. Karger AG, Basel

\section{KARGER}

Fax +4161306 1234 E-Mail karger@karger.ch www.karger.com
(C) 2010 S. Karger AG, Basel www.karger.com/sfn

\section{Introduction}

The stereotactic frame has been the method of choice for the accurate placement of electrodes in functional neurosurgery since its first use on humans some 60 years ago [1]. It provides guidance to a neurosurgical target by means of a fiducial system, and it provides a steady, adjustable holder for a probe as it is advanced toward the target. With the FDA (Food and Drug Administration) approval of deep brain stimulation (DBS) for the treatment of movement disorders, the placement of active electrodes has become a standard treatment for patients who do not respond to medication or cannot tolerate its side effects, and the vast majority of placements are performed with traditional frames such as the Leksell G frame or the Cosman-Roberts-Wells (CRW) frame [2, 3]. Deep brain stimulators have the potential to relieve symptoms with minimal side effects, but accuracy is critical because of the small size of the target structures. The size of the subthalamic nucleus (STN), for example, is of the order of $6 \times 4 \times 5 \mathrm{~mm}$ [4], and a placement error of 3-4 millimeters can result in unsatisfactory results [5], either because of low effectiveness and shortened battery life resulting from the need for increased current, or because of considerable side effects. The accuracy of the frame is an important factor in successful electrode placement. To 
measure this accuracy, we define the electrode placement error (EPE) as the distance between the calculated intraoperative position of the implant relative to the stereotactic frame and the position of this implant relative to the frame as observed in postoperative CT images (more details on the technique we used to compute the EPE are provided in the Materials and Methods section). In the remainder of this article, the intraoperative position of the implant provided by the frame will be called the final electrode position (FEP).

In a 2005 study of both the CRW frame (Radionics, Burlington, Mass., USA) and the Leksell G frame (Elekta AB, Stockholm, Sweden), Holloway et al. [6], measured the EPE and found a mean of $3.2 \pm 1.4 \mathrm{~mm}$. In a 2007 intraoperative study where implantation was guided by intraoperative imaging and the implant position was extracted using intraoperative stereotactic X-ray immediately after implantation, Bjartmarz and Rehncrona [7] measured a mean EPE of $1.2 \pm 0.6 \mathrm{~mm}$ for the $\mathrm{G}$ frame.

The widespread use of traditional frames like the CRW and Leksell G shows clearly that they serve as feasible approaches for DBS surgery. However, they have notable drawbacks. Firstly, because of their size and weight and because they surround the head, they are uncomfortable for the patient before surgery, and during surgery they reduce the freedom of motion of the patient, who is often awake and must interact with a neurophysiologist during the procedure. Secondly, they are not readily adapted to bilateral insertions in which 2 separate cannulae are placed simultaneously. To address these problems, 2 alternative solutions have recently been introduced; the NexFrame (Medtronic Inc., Minneapolis, Minn., USA) and the StarFix microTargeting ${ }^{\circledR}$ Platform (FHC Inc., Bowdoin, Me., USA).

The NexFrame is a total departure from the traditional frame even though like the traditional frame, it is fixed directly to the skull and can be adjusted in order to aim a probe at a target. One difference is that it is much smaller and lighter, but its major difference is that, unlike the traditional frame, it provides no navigational guidance. Instead, guidance must be provided by an ancillary tracking system such as the Stealth system (Medtronic SNT, Louisville, Colo., USA). The 2005 study by Holloway et al. [6] also measured EPE for the NexFrame and found a mean of $3.2 \pm 1.4 \mathrm{~mm}$ (coincidentally the same statistics as for the stereotactic frames). Similarly, in their 2007 study, Bjartmarz and Rehncrona [7] evaluated the NexFrame and found an intraoperative EPE of $2.5 \pm 1.4 \mathrm{~mm}$.

Like the NexFrame, the StarFix platform is fixed to the skull and is much smaller and lighter than the tradition- al frame, but the StarFix, unlike the NexFrame, provides its own navigational guidance. No ancillary tracking system is required. Its major functional difference from the frame (and the NexFrame) is that it requires no intraoperative adjustment in order to aim a cannula at a target. Instead, the StarFix platform is custom made for each patient and each target so that, when the platform is fixed to the patient, a driver mounted on the platform and a cannula inserted, the cannula is automatically aimed at the target. Adjustments can be made intraoperatively, but they are unnecessary unless the position of the target relative to the skull is changed intraoperatively. Furthermore, the platform can be made such that 2 separate, preaimed cannulae can be advanced with 2 separate drivers toward dual targets with a single platform. A 2005 study by some of us using the same method used in this paper but without accounting for brain shift found the EPE for the StarFix platform to be $2.8 \pm 0.8 \mathrm{~mm}$ [8].

Phantom studies have been carried out for these systems as well, and, not surprisingly, the measured EPE tend to be smaller than those measured intraoperatively. In 1994, Maciunas et al. [9] found an EPE of $1.8 \pm 1.1 \mathrm{~mm}$ for the CRW frame and $1.7 \pm 1.0 \mathrm{~mm}$ for the Leksell G frame. In 2004, Henderson et al. [10] measured an EPE for the NexFrame of $1.25 \pm 0.6 \mathrm{~mm}$. In a 2008 study, some of us found an EPE for the StarFix platform of 0.42 $\pm 0.15 \mathrm{~mm}$ [11].

Including both clinical and phantom studies, these errors represent a wide range: $1.2-3.2 \mathrm{~mm}$ for the traditional frames, $1.25-3.2 \mathrm{~mm}$ for the NexFrame and 0.42-2.8 $\mathrm{mm}$ for the StarFix platform. The explanation may lie in the measurement techniques. An examination of the descriptions of the techniques reveals that in every study reporting a mean EPE greater than $2.5 \mathrm{~mm}$, the EPE were measured by comparing lead positions based on the stereotactic platform and microdrive system with the actual lead positions extracted from postoperative CT. With this method, the measurement of the EPE is confounded by movement of the brain between electrode placement and postoperative imaging. Such motion is to be expected because of air invasion due to negative pressure inside the brain and/or the draining of cerebrospinal fluid (CSF), swelling during the surgery and/or some partial resolution of these effects by the time of postoperative imaging. The one clinical study to date in which the effect of brain shift was avoided is that of Bjartmarz and Rehncrona [7], who used intraoperative crossed-beam X-ray imaging. Their smaller means $(1.2 \mathrm{~mm}$ for the $\mathrm{G}$ frame and $2.5 \mathrm{~mm}$ for the NexFrame) suggest that brain shift may have caused an overestimate of EPE in the other clinical studies. 
In their respective studies, Flickinger et al. and Ashpole et al. have shown that EPE measurement can be affected by 2 other errors: human error and error due to the flexible nature of the electrode. Flickinger et al. [12] report that errors in the adjustment of the stereotactic frame can be made in up to $12 \%$ of the cases and suggested that coordinates should be verified by a minimum of 2 observers. Ashpole et al. [13] have discussed how placement errors occur because of the flexible nature of the final implant and have suggested the use of an instrument which is a hollow yet sturdy device through which the final implant can be inserted to be left in position before this instrument is withdrawn.

In this paper, we describe a retrospective analysis of data acquired at our institution via comparison of intraand postoperative lead positions to analyze the effect of brain shift on EPE estimation. We present evidence supporting the thesis that the occurrence of brain shift does indeed result in a significant overestimate of EPE, and we show how to estimate true EPE by analyzing the data in terms of the magnitude of shift.

\section{Materials and Methods}

\section{Subjects}

With institutional review board (Vanderbilt University IRB No. 060232) approval we retrospectively analyzed 75 patients who underwent DBS surgery from 2002 to 2008 . Unilateral (5 patients) or bilateral (70 patients) implant placements in the globus pallidus internus (8 patients), STN (49 patients) and ventral intermediate nucleus (18 patients) were included in the study. This dataset comprises 45 male and 30 female subjects. The mean age was $60 \pm 12$ years. Two neurosurgeons, coauthors P.E.K. and J.S.N., performed surgeries on 41 and 34 patients, respectively.

\section{Surgical Procedure and Imaging Sequences}

Functional neurosurgical procedures such as DBS require precise targeting of small areas deep inside the brain. Traditionally, this involves the preoperative selection of an approximate target location (henceforth referred as preoperative target position), followed by intraoperative adjustments using signal recordings and stimulations. At our institution, targets are first selected using an automatic method presented by D'Haese et al. [14], then refined and validated visually by the surgeon using the patient's MRI scan. Traditionally, the patient undergoes a preoperative CT and MRI scans. CT images are acquired at $\mathrm{kVp}=120 \mathrm{~V}$, exposure $=$ $350 \mathrm{mAs}, 512 \times 512$ pixels. The in-plane resolution ranges from 0.42 to $0.62 \mathrm{~mm}$, and the slice thickness from 0.75 to $1 \mathrm{~mm}$. MRI images are 3-D volumes (TR: $12.2 \mathrm{~ms}$; TE: $2.4 \mathrm{~ms} ; 256 \times 256 \times$ 170 voxels, with typical voxel resolution of $1 \times 1 \times 1 \mathrm{~mm}) \mathrm{ac}-$ quired using the SENSE parallel imaging technique $\left(\mathrm{T}_{1}\right.$-weighted, 3-D, turbo field echo MRI from Philips) on a 3-tesla scanner.

At the time of surgery, the patient is placed in a sitting position, burr holes are drilled, the dura is opened, and a stereotactic cus-

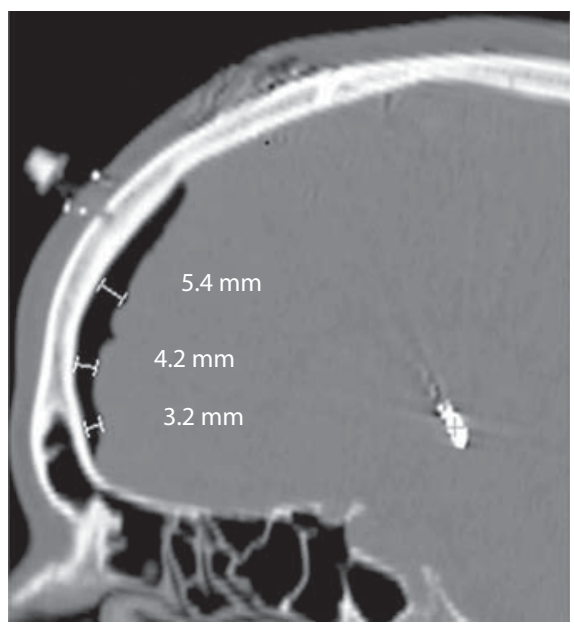

Fig. 1. Typical medium pneumocephalus in a sagittal slice of a postoperative CT scan for a patient who underwent DBS. This patient was classified into the medium brain movement category. Widths of the pneumocephalus measured at 3 positions are shown. Gray cross: center of the implant.

tom-madeStarFix microTargetingPlatform [501(K), No. K003776], manufactured using the patient's preoperative CT scan and planned target data, is affixed to the patient's head. Next, a microdrive is affixed to the frame, and 3 or 4 parallel cannulae used as guides for recording and stimulating electrodes are inserted. After physiological target localization using microelectrode recording and stimulation, the DBS electrode (typically model No. 3389 for STN and No. 3387 for globus pallidus internus and ventral intermediate nucleus; Medtronic Inc.) is implanted using a rigid stylet to guide the lead to the chosen location and avoid errors discussed by Ashpole et al. [13]. This is done by removing only 1 cannula and replacing it by the stylet. Once the implant is in place, the stylet and the other cannulae are removed.

An error can be made during the placement of the lead. This error is the sum of the mechanical error of the frame and microdrive system and human error. To minimize human error, all settings are checked by a minimum of 2 persons as suggested by Flickinger et al. [12]. Postoperatively, on the same day, the patient is transferred to a CT imaging unit in supine position (to minimize brain movement). A postoperative CT is obtained to confirm the lead position in the skull and check for pneumocephalus. Figure 1 shows a typical postoperative CT image with intracranial air. From 1 week to 1 month after surgery, the intracranial air resolves, and the patient undergoes another postoperative CT scan to infer the final stabilized position of the lead within the brain.

\section{Intraoperative Placement Error Estimation}

When the cannulae and stylet that were holding the brain while placing the lead are removed, the brain is free to move within the skull. This is illustrated in figure 2 . The lead can be expected to move with the brain since it is no longer rigidly held in position. Therefore, by comparing the intraoperative FEP provided by 
Fig. 2. a The cannulae are in place and restrict the brain's movement in their vicinity. b After the recordings and stimulation, the implant is placed at the optimal position (FEP) using a stiff stylet. Gray anterior region: small pneumocephalus. c The stylet and cannulae are removed, and the brain shifts and the lead shifts with it. The larger gray region shows that the pneumocephalus has grown as a result of the shift.

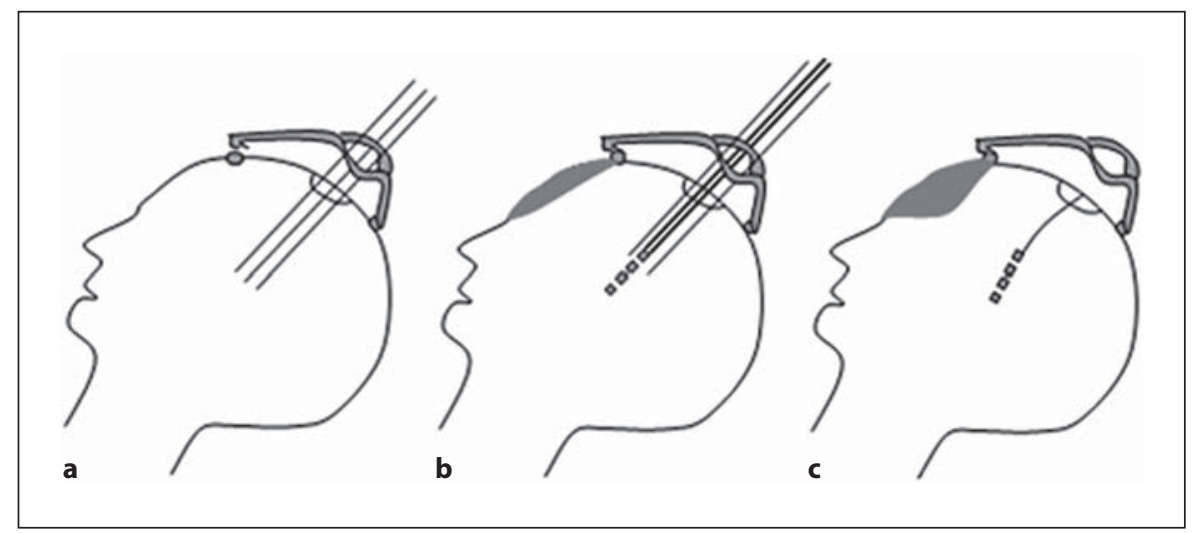

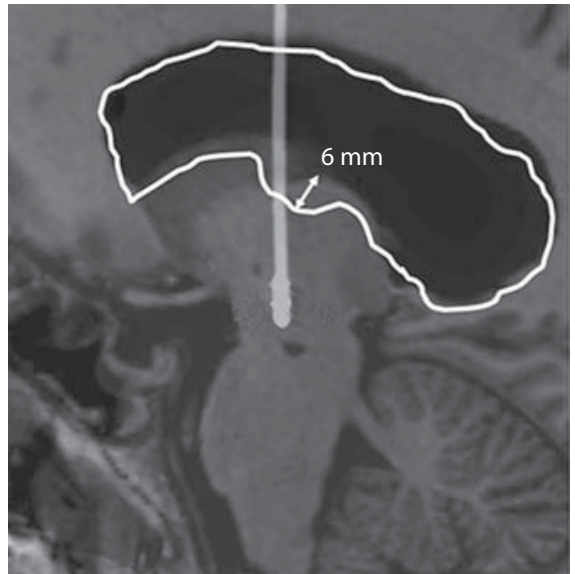

Fig. 3. Movement around the ventricles due to CSF changes during DBS. The segmentation of the ventricle from a postoperative CT slice is overlaid as a white contour on the corresponding slice in the preoperative MRI (sagittal slice). This patient showed no pneumocephalus but still had $>6 \mathrm{~mm}$ shift around the ventricles due to CSF leakage. The nearly vertical light gray stripe is the implanted electrode lead.

the frame with the position of the lead extracted from the postoperative scan, we measure not only the EPE but also the amount of brain shift that happens after the lead placement. In this study, we propose to isolate the EPE from brain shift by selecting patients that show minimal pneumocephalus on their postoperative scans.

The postoperative scans of the 75 patients were carefully inspected and classified into 3 categories depending on the amount of brain movement: low, medium and large. This was based on the amount of air invasion between the frontal lobe and the skull. To estimate the amount of such brain shift, we calculate the average width of the air pocket between the cortical surface and the skull on a sagittal slice aligned to the anterior commissure-posterior commissure reference as shown in figure 2 . A measure of $<3 \mathrm{~mm}$ is considered as a low, between 3 and $7 \mathrm{~mm}$ as a medium, and
Table 1. Summary of the rules used to classify each lead into movement categories

\begin{tabular}{lll}
\hline Movement & Pneumocephalus & Ventricles \\
\hline Low & $\leq 3 \mathrm{~mm}$ & $\leq 1 \mathrm{~mm}$ \\
Medium & $>3$ and $\leq 7 \mathrm{~mm}$ & $>1$ and $\leq 3 \mathrm{~mm}$ \\
Large & $>7 \mathrm{~mm}$ & $>3 \mathrm{~mm}$ \\
\hline
\end{tabular}

$>7 \mathrm{~mm}$ as a large amount of air. This is done independently for both sides.

Some cases might show low pneumocephalus but still provide evidence of large brain displacement. This has been found in certain cases with very large ventricles as shown in figure 3. Such cases are then classified based on the displacement of the ventricles measured at the level of the lead. A ventricular displacement of $\leq 1 \mathrm{~mm}$ is considered as low, between 1 and $3 \mathrm{~mm}$ as medium, and $>3 \mathrm{~mm}$ as large movement. Table 1 summarizes the classification rules for the 3 categories.

In the cases categorized as having low expected brain shift, the distance between the FEP and the postoperative lead position provides a good estimate of the EPE. This is done as follows. Like all intraoperative observations, the FEP position is recorded intraoperatively relative to the system of reference of the StarFix platform (e.g. 'center track, $2 \mathrm{~mm}$ above target'). That platform is affixed to the head of the patient using bone markers. Because the geometry of the platform is known and markers are visible in the preoperative CT, the FEP position can be translated into $x, y$ and $z$ coordinates in the frame of reference of the preoperative CT. By means of image registration between the post- and preoperative CT scans, it is also possible to transform the observed postoperative position of the implant into the system of reference of the preoperative CT. Thus, both the location of the FEP as read from the StarFix microdrive and the actual implant position obtained from the postoperative CT are now in the same space and can be easily compared. As explained in the Introduction, the EPE is defined as the distance between these 2 positions. The actual position of the electrode is extracted from the postoperative CT using the electrode localization algorithm described in the next section. 
Fig. 4. a Various components of our lead extraction algorithm. A box is defined around the intraoperative FEP to isolate the lead. From inferior to superior, each slice of the box (dashed horizontal line) is then scanned for an intersection with the lead. Once found, the center of gravity of the intersection is computed. Dark segment: intersection of slice $i$ with the lead; triangle: center of gravity of the lead. All the centers of gravity are then used to compute the orientation of the lead. Knowing the slice that first intersected the lead, and the dimensions and orientation of the implant, the coordinates of the midpoint of the electrode are calculated (double circle). The displacement from the FEP to the midpoint is the estimated EPE. b A typical lead extraction result using this method.

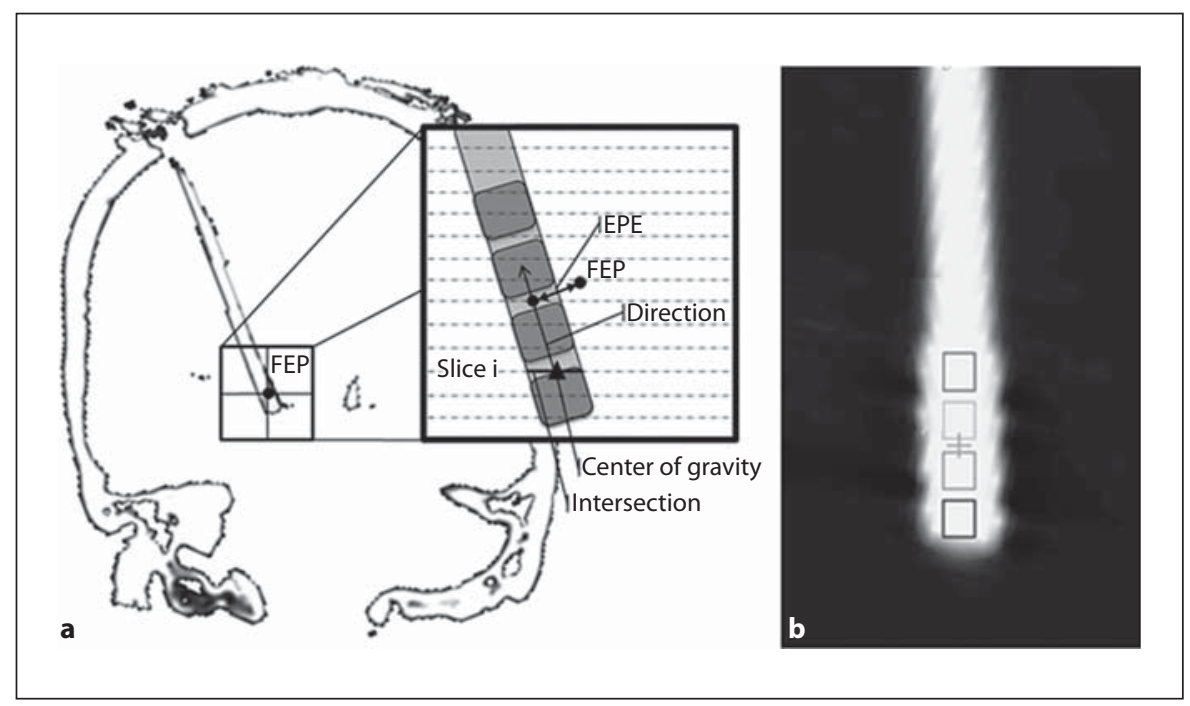

\section{Automatic Lead Extraction}

We developed a method to automatically extract the position of the leads in the postoperative CT scans. In order to map the lead coordinates into the preoperative CT space, the postoperative CT scans are first rigidly aligned to the preoperative CT using mutual-information-based image registration [15].

Figure 4a shows the various components of the extraction algorithm, and figure $4 \mathrm{~b}$ shows a typical extraction. After applying a threshold to the aligned images to reduce noise and artifacts, the algorithm selects a bounding box $\left(25 \mathrm{~mm}^{3}\right)$ centered on the FEP and works within that box. This is done to get rid of additional artifacts caused by the presence of bone markers or additional leads. From inferior to superior, each slice of the box is scanned for an intersection with the lead. Once found, the center of gravity of the intersection is computed and recorded for each slice. Each of these centers of gravity is used to find the orientation of the lead. Finally, knowing the slice that first intersected the lead and the dimensions and orientation of the implant, the coordinates of the midpoint of the electrode are computed. Bad images (movement during acquisition or reconstruction problem), inaccurate registrations or the presence of artifacts can cause the automatic extraction method to fail. Therefore, all localizations are manually verified and adjusted when required.

\section{Results}

\section{Exclusions}

Each case was manually reviewed to make sure it satisfied the selection criteria. We excluded 46 of the 150 leads that were available for the study. Leads were excluded if: (1) more than 1 lead was placed on the same side of the brain causing excessive artifacts (2 leads); (2) the pre- or postoperative CT was of poor quality or showed motion artifacts (27 leads); (3) the postoperative CT was not acquired on the day of the surgery (3 leads), or (4) the FEP was not recorded (14 leads).

\section{Electrode Displacement}

Based on the approach described earlier, each lead was categorized. This resulted in 31 leads in the low, 42 in the medium and 31 in the large movement category (table 2).

Table 3 reports the means $\pm \mathrm{SD}$ of the distance between the FEP and the actual lead position for each category. The 3 categories present statistically different $(\mathrm{p}<$ 0.01 ) results: $1.24 \pm 0.37 \mathrm{~mm}, 2.20 \pm 0.70 \mathrm{~mm}$ and 2.56 $\pm 1.08 \mathrm{~mm}$, respectively, for the low, medium and large movement groups. The same statistic computed using all the 104 leads (combining groups) is $1.99 \pm 0.92 \mathrm{~mm}$. For the low movement group, we further decomposed the error into 1 component parallel and 1 component perpendicular to the trajectory. The statistics were $0.56 \pm 0.4$ $\mathrm{mm}$ for the parallel component and $1.0 \pm 0.6 \mathrm{~mm}$ for the perpendicular component.

\section{Discussion}

The outcome of our analysis corroborates the findings of Bjartmarz and Rehncron [7] in their study in which the effect of brain shift was avoided. Our results indicate that the EPE was overestimated at least by about 60\%, from $1.24 \pm 0.37 \mathrm{~mm}$ to $1.99 \pm 0.92 \mathrm{~mm}$, when brain shift was not taken into account. We have presented a strategy to 
Table 2. Details about the number of leads excluded and used for each of the 3 movement categories

\begin{tabular}{lclc}
\hline Movement & All & Exclusions & Used \\
\hline Large & 54 & 23 & 31 \\
Medium & 54 & 12 & 42 \\
Low & 42 & 11 & 31 \\
\hline Total & 150 & 46 & 104 \\
\hline
\end{tabular}

Table 3. Means and SD of the distance between the FEP and the actual lead position for each movement category

\begin{tabular}{llll}
\hline Movement & Means & SD & Count, $\mathrm{n}$ \\
\hline Low & 1.24 & 0.37 & 31 \\
Medium & 2.20 & 0.70 & 42 \\
Large & 2.56 & 1.08 & 31 \\
\hline All $^{1}$ & 1.99 & 0.92 & 104 \\
\hline
\end{tabular}

${ }^{1}$ Computed using all the leads without brain movement distinction.

minimize the effect of brain shift on the estimation of the EPE for a stereotactic platform in the absence of intraoperative imaging data, and we have applied it to the StarFix microTargeting Platform. Based on our analysis, using the StarFix frame clinically, we estimate that the EPE, which includes both frame and human error, is $\leq 1.24 \pm$ $0.37 \mathrm{~mm}$. This accuracy is comparable to that reported for the $G$ frame $(1.2 \mathrm{~mm})$, and better than that reported for the Nexframe $(2.5 \mathrm{~mm})$ [7]. There is an additional er- ror that affects EPE, which is the error due to the fixation of the implant to the skull. The main component of this error can be expected to be along the trajectory. Because the parallel component of the error that we have measured is smaller than the perpendicular component, our results suggest that this is not a major source of error, but it is nevertheless included in our frame accuracy measurements.

Also, between the placement of the implant and the acquisition of postoperative CT, despite all efforts to keep the patient supine and stationary, the patient's head orientation may change somewhat leading to gravity-induced brain shift, causing the lead to shift with it. Even for cases in the low movement category, this shift may not be completely absent and may still cause some overestimation of EPE.

The results we obtained indicate that the brain shifts after the cannulae have been removed. This suggests that these cannulae hold the brain in place while the procedure is ongoing. Our findings also suggest that brain shift does not happen instantaneously upon the opening of the dura, but progresses throughout the surgery; when possible, a cannula should be kept in the brain when alternate tracks are being explored. These findings have recently been corroborated, albeit on a small number of patients, with intraoperative CT scans obtained after the opening of the dura, after the placement of the DBS lead with the cannulae in place, and after the removal of the cannulae [16].

\section{Acknowledgment}

This research has been supported, in parts, by NIH grant R01EB0006136.

\section{References}

1 Spiegel EA, Wycis HT, Marks M, Lee AL: Stereotaxic apparatus for operation of the human brain. Science 1947;106:349-350.

-2 Benabid AL, Vercucil L, Benazzouz A, Koudsie A, Chabardes S, Minotti L, Kahane P, Gentil M, Lenartz D, Andressen C, Krack P, Pollak P: Deep brain stimulation: what does it offer? Adv Neurol 2003;91:293-302.

3 Deuschl G, Wenzelburger R, Kopper F, Volkmann J: Deep brain stimulation of the subthalamic nucleus for Parkinson's disease: a therapy approaching evidence-based standards. J Neurol 2003;250(suppl 1):143-146.
Richter EO, Hoque T, Halliday W, Lozano AM, Saint-Cyr JA: Determining the position and size of the subthalamic nucleus based on magnetic resonance imaging results in patients with advanced Parkinson disease. J Neurosurg 2004;100:541-546.

5 Schrader B, Hamel W, Weinert D, Mehdorn HM: Documentation of electrode localization. Mov Disord 2002;17(suppl 3):S167S174.
Holloway KL, Gaede SE, Starr PA, Rosenow JM, Ramakrishnan V, Henderson JM: Frameless stereotaxy using bone fiducial markers for deep brain stimulation. J Neurosurg 2005;103:404-413.

7 Bjartmarz H, Rehncrona S: Comparison of accuracy and precision between frame-based and frameless stereotactic navigation for deep brain stimulation electrode implantation. Stereotact Funct Neurosurg 2007;85: 235-242. 
8 Fitzpatrick JM, Konrad PE, Nickele C, Cetinkaya E: Accuracy of customized miniature stereotactic platforms. Stereotact Funct Neurosurg 2005;83:25-31.

$\checkmark 9$ Maciunas RJ, Galloway RL, Latimer JW: The application accuracy of stereotactic frames. Neurosurgery 1994;35:682-694.

-10 Henderson JM, Holloway KL, Gaede SE, Rosenow JM: The application accuracy of a skull-mounted trajectory guide for imageguided functional neurosurgery. Comput Aided Surg 2004;9:155-160.
Balachandran R, Mitchell JE, Dawant BM, Fitzpatrick JM: Accuracy evaluation of microTargeting Platforms for deep-brain stimulation using virtual targets. IEEE Trans Biomed Eng 2009;56:37-44.

12 Flickinger JC, Lunsford LD, Kondziolka D, Maitz A: Potential human error in setting stereotactic coordinates for radiosurgery: implication for quality assurance. Int J Radiat Oncol Biol Phys 1993;27:397-401.

13 Ashpole RD, Fabinyi GC, Vosmansky M: A new instrument for improved accuracy of stereotactic depth electrode placement: technical note. J Neurosurg 1996;85:357-358.

-14 D'Haese P-F, Cetinkaya E, Konrad PE, Kao C, Dawant BM: Computer-aided placement of deep brain stimulators: from planning to intraoperative guidance. IEEE Trans Med Imaging 2005;24:1469-1478.
5 Maes F, Collignon A, Vandermeulen D, Marchal G, Suetens P: Multimodality image registration by maximization of mutual information. IEEE Trans Med Imaging 1997; 16:187-198.

16 Remple MS, Kao C, D'Haese P-F, Pallavaram S, Konrad PE, Neimat JS, Franck JI, Dawant BM: Lessons learned from the creation of an electrophysiological atlas for deep brain stimulation (DBS). American Society of Neurophysiological Monitoring annual meeting, Special interest group presentation, Vancouver, 2009. 